\title{
The European Association of Clinical Psychology and Psychological Treatment (EACLIPT): A New Organization for the Future!
}

\author{
Gerhard Andersson ${ }^{\mathrm{ab}}$ \\ [a] Department of Behavioural Sciences and Learning, Linköping University, Linköping, Sweden. [b] Department of \\ Clinical Neuroscience, Karolinska Institute, Stockholm, Sweden.
}

Clinical Psychology in Europe, 2019, Vol. 1(1), Article e33241, https://doi.org/10.32872/cpe.v1i1.33241

Published (VoR): 2019-03-29

Corresponding Author: Gerhard Andersson, Department of Clinical Neuroscience (CNS), K8, CPF - KCP, Liljeholmstorget 7b Plan 611763 Stockholm, Sweden. E-mail: gerhard.andersson@liu.se

The European Association of Clinical Psychology and Psychological Treatment (EACLIPT) was founded in 2017 with representatives of many European countries. At its launch, many people were surprised to hear that such an organization did not already exist given the role of clinical psychology both as a branch of psychology and psychological research, but also as a renowned profession.

We knew several national organizations existed for clinical psychologist practitioners and researchers across Europe, as well as European and international organizations for various subdisciplines of psychology and forms of psychotherapy. But we regarded the absence of a targeted organization for Europe as a serious omission. Thus, the EACLIPT aims to strengthen science, practice and political representation in relation to clinical psychology.

In this editorial we will briefly describe the aims of the EACLIPT and also our achievements to date. Finally, we will outline our wishes for the future.

The EACLIPT's aims are broad. We want to foster research, education and dissemination of scientifically evaluated findings, and address the following topics:

- Diagnostics and classification of mental health conditions

- Psychological and psychobiological mechanisms of health and disease

- Psychological treatments, psychotherapy

- Prevention and rehabilitation

- Healthcare issues in mental health conditions 
- Dissemination and implementation of evidence-based psychological treatments

- Education in clinical psychology

- Representation of clinical psychology in politics across Europe.

Although the EACLIPT focuses on clinical psychology, we are also dedicated to both research and practice. It is, however, by no means an organization exclusively for clinicians, since we also have a strong interest in the status of clinical psychology as a research area and as an important profession from a policy perspective. Thus, policy and research are regarded as more urgent areas for EACLIPT to focus on than the actual practice of clinical psychology. The latter has many national and international organizations and, when it comes to psychotherapy brands, also several psychotherapy organizations.

The EACLIPT is needed right now for several reasons. First, we believe that clinical psychology is more than psychotherapy. Second, the world, and indeed Europe, is shrinking as practitioners and patients move across borders. This requires European-wide standards both in research and in clinical practice, and also cross-border collaboration. The profession of clinical psychology is also expanding into medicine and healthcare in general. This necessitates research into disorders and health problems that have often been regarded as extraneous to psychology. Good practice in research and clinical tasks demand that we define quality criteria for training in and provision of clinical psychological healthcare, and that we improve comparability of training programs in European countries.

What have we done so far? Following our initial gathering in Amsterdam, the Netherlands, in 2017, we arranged a small closed inaugural conference in Linköping, Sweden, in 2018. In between those two meetings we formed a board which then convened in Amsterdam. We also had regular monthly board meetings by phone and developed a website www.eaclipt.org. And, as you can see, the journal was initiated and launched its first issue in 2019.

The board has also actively sought to recruit members, find national representatives and attend important meetings at EU-level (e.g., parliament). We have also had to deal with numerous practical matters that accompany an organization's launch. We have also initiated a newsletter. Although the exact number of our members is unknown at this editorial's publication, membership topped 400 at the time of writing. We have also began planning for our first conference. The first European congress of the EACLIPT will be held in Germany, Dresden, 31 Oct - 02 Nov 2019, under the topic: "No health without mental health: European clinical psychology takes responsibility".

Finally we should mention our expectations. We hope that the EACLIPT will help advancing the field of clinical psychology and all connected academic and clinical fields in Europe. We expect clinical psychology to be even more relevant in the future than it is today. Clinical psychology has already had a favourable impact on the treatment of mental health conditions and, increasingly, other health problems too. This has been driven 
by progress in research. But it is not enough just to know what works when it comes to clinical problems (including both assessment and treatment procedures). We also need to make an effort to disseminate that knowledge, not least at policy level. Finally, we hope that the profession of both researchers and clinicians (sometimes the same person serving in both functions) will benefit from the EACLIPT and that we will manage to develop policy documents and research collaborations across Europe.

Gerhard Andersson, president EACLIPT

Further Board members: Claudi Bockting, Roman Cieślak, Céline Douillez, Thomas Ehring, Andreas Maercker, Winfried Rief 